\title{
Wave-Particle-Collisional Planetary-Black-Hole Formation
}

\author{
By Rodney Bartlett
}

\begin{abstract}
-
This Letter was inspired by the Exoplanet section of an Astrophysics course conducted by the Australian National University on the educational site edX - specifically, a video presented by Dr. Paul Francis and Nobel Physics Laureate Brian Schmidt which asks, Why don't asteroids fuse together to form planets? From this starting point is developed a hypothesis explaining planetary, and black hole, formation not in terms of collisions but as a function of geometry that traces its roots to Einstein's paper "Do gravitational fields play an essential role in the structure of elementary particles?" The geometry embraces the mass and quantum spin of particles of matter, the Higgs boson, plus the bosons of the weak and strong nuclear forces.
\end{abstract}

\section{PROTOPLANETARY MERGERS?}

Why don't asteroids fuse together to form planets? The video "Collisional Dust Formation" (https://courses.edx.org/courses/course-v1:ANUx+ANUASTRO2x+2T2016/courseware/4e44621a1e3e4aee9e7cc6546274029d/cb898dc8ad1b 44a2aecc1e10ba2a0669/11 ?activate block id=block-v1\%3AANUx\%2BANUASTRO2x\%2B2T2016\%2Btype\%40html\%2Bblock\%40c250bfc6215a43418fdfe465390f $\underline{0693}$ ) says they could come "... gently together, until they merge and form big things. As they did collide, it'd be a very gentle collision, just like two pillows gently merging together and so it would make something bigger." If we cling to the idea of planets being formed from mergers between asteroids and other debris, this is probably the only scenario we can devise. But I don't see how mergers of any kind can solve this question. If asteroids crash together, you get dust. If they come together gently, you do indeed get something bigger, but it isn't the beginnings of one planet - it's two asteroids that may be in firm contact but are nevertheless separate. Consider this article in the journal "Astronomy \& Astrophysics" - "Why do protoplanetary disks appear not massive enough to form the known exoplanet population?" (October 2018 https://doi.org/10.1051/0004-6361/201834076) That article suggests, "An extremely efficient recycling of dust particles in the disk cannot solve this conundrum. This implies that either the cores of planets have formed very rapidly $(<0.1-1 \mathrm{Myr})$ and a large amount of gas is expelled on the same timescales from the disk, or that disks are continuously replenished by fresh planet-forming material from the environment." 
As far as I can tell, another plausible explanation is the following. My hypothesis takes a fresh look at planetary formation by 1) removing the topic of mergers, but not of gas and dust, and 2) requiring people to do nothing more than think about the title of a 1919 paper by Albert Einstein, "Do gravitational fields play an essential role in the structure of elementary particles?" (he published this four years after his famous General Relativity, so it seems very possible that the question posed by the title reveals that Einstein was even ahead of our time when it comes to defining mass)

\section{GEOMETRY, MASS, SPIN}

The concept of radiation pressure can be applied to what I call "vector-tensor-scalar geometry" because "the energy and momentum of the photons and presently hypothetical gravitons exert a pressure we call mass". Two adjoining sides of a parallelogram represent the vectors of the photon's spin 1 and the graviton's spin 2 . The resultant diagonal represents the interaction of the sides/vectors $(1 \div 2=$ the spin $1 / 2$ of every matter particle). Tensor calculus changes the coordinates of the sides into the coordinates of a position on a line (a single point on the diagonal). This scalar point is associated with particles of spin 0 . If the mass produced during the $1 \div 2$ interaction - the energy and momentum of the photons and presently hypothetical gravitons exert a pressure we call mass - happens to be $125 \mathrm{GeV} / \mathrm{c} 2$, its union with spin 0 produces the Higgs boson. $125 \mathrm{GeV} / \mathrm{c} 2$ united with spin 0 means the central scalar point of the Higgs boson is related to the vector of the graviton's spin 2, and the Higgs field is therefore united with the supposedly unrelated gravitational field (together with the latter's constant interaction with the electromagnetic field).

Stress may be defined, in this case, as a physical quantity that expresses the continuous internal pressure that photon and graviton particles exert during the thermodynamical process of formation of properties of matter. Combining this with tensor calculus changing the coordinates of the sides into the coordinates of a position on a line (the diagonal) could change the term "vector-tensor-scalar geometry" into the term "stress tensor". The geometry's two adjoining sides of a parallelogram may be thought of as eigenvectors ("eigen" is a German word meaning "characteristic") of a transforming, nonzero nature that produce the eigenvalues of the diagonal, which include the scalar Higgs boson as well as other values such as the spin 1 bosons of the strong/weak nuclear forces. 
It must be remembered that referring to space alone is incomplete. Living in space-time, it's necessary to add some sentences about the time factor. The photon must interact with the graviton to produce the mass of the weak nuclear force's $W$ and $Z$ bosons. To produce their quantum spin, the photon's spin 1 needs to react with the graviton's spin 2. That is, the photon's turning through one complete revolution needs to be combined with the graviton's being turned through two half-revolutions. ${ }^{\wedge}$ Incorporating the time factor as a reversal of time in the middle of the interaction: a gravitonic half revolution is subtracted from the photonic full revolution then the graviton's time-reversal adds a half revolution $(1-1 / 2+1 / 2$ = the spin 1 of $W$ and $Z$ bosons). The strong nuclear force's gluon's quantum spin of 1 could arise in the same way as the spin 1 of weak-force bosons.

^ Professor Hawking writes, "What the spin of a particle really tells us is what the particle looks like from different directions." (Stephen Hawking, "A Brief History of Time"

- Bantam Press, 1988)

Spin 1 is like an arrow-tip pointing, say, up. A photon has to be turned round a full revolution of 360 degrees to look the same.

Spin 2 is like an arrow with 2 tips - 1 pointing up, 1 down. A graviton has to be turned half a revolution (180 degrees) to look the same.

Spin 0 is like a ball of arrows having no spaces. A Higgs boson looks like a dot: the same from every direction.

Spin $1 / 2$ is logically like a Mobius strip, though Hawking doesn't specifically say so. This is because a particle of matter has to be turned through two complete revolutions to look the same, and you must travel around a Mobius strip twice to reach the starting point.

\section{PPD, BLACK HOLES, NUCLEAR BOSONS}

The interacting gravity and electromagnetism produce mass e.g. they can form a Higgs boson or the weak nuclear force's bosons as well as matter. On a cosmic level - if gravitational and electromagnetic waves scan a protoplanetary disk surrounding a newborn star, the quantum spin of the particles of matter in the disk (1 / 2) could imprint $^{\wedge}$ itself on the waves' interaction and build up a planet layer by layer from vectortensor-scalar geometry's $1 \div 2$ interaction. This method of building planets is preferred to collisions between rocks and dust in the disk because most planetary systems seem to outweigh the protoplanetary disks in which they formed, leaving astronomers to reevaluate planet-formation theories. If the waves focus on a region of space where there's no matter, the opposite interaction occurs and the graviton's spin 2 is divided by the photon's spin 1 to produce $2 \div 1$. The mass produced has the spin inherent in each of 
the gravitons composing the body of gravitational waves, and could be an alternative method to supernovas for making black holes \& their great gravity (space-time curves).

^ What do I mean by "imprint"? To explain using something different - when a laser scanner reads a barcode, the information in the barcode is "imprinted" in the scanner. When gravitational and electromagnetic waves scan a protoplanetary disk, the disk acts as a "seed" and its info is recorded or imprinted in the waves. When they scan "empty" space-time, the info pertaining to the graviton's spin of $2(2 / 1)$ is imprinted since gravity is space-time according to General Relativity: it says gravity is just another term for the curvature of space-time. A black hole would be a focus of gravitational waves that is devoid of "seed" material and isn't capable of making physical astronomical bodies. If the waves focus on a region of space where there's no matter, the opposite interaction occurs and the graviton's spin 2 is divided by the photon's spin 1 to produce $2 \div 1$. The mass produced has the spin inherent in a body of gravitational waves, and could be an alternative method to supernovas for producing black holes since General Relativity says gravity is a push caused by the curvature of space-time. The increased gravitation associated with Sagittarius $A^{*}$, the supermassive black hole at the centre of our galaxy, may enable layer-by-layer construction affecting stars that will orbit Sagittarius $A^{*}$ within approximately 30,000 light years to be "top heavy", and to produce more massive stars in preference to low-mass ones. ("The dependence of star formation on initial conditions and molecular cloud structure" - Monthly Notices of the Royal Astronomical Society, Volume 397, Issue 1, Pages 232-248, https://doi.org/10.1111/j.13652966.2009.14970.x)

This top-heaviness could result from increased scanning strength of both the gravitational and electromagnetic vectors in the geometry above (from the enormous gravity associated with Sgr $\mathrm{A}^{*}$ - and from the visible light, heat, X-rays etc associated with the accretion disk orbiting the supermassive black hole). The quantum spins of the photon and hypothetical graviton could produce the quantum spin of particles of matter via their $1 / 2$ interaction. Via their energy and momentum, photon and graviton could produce what we call the mass of matter particles. Using $\mathrm{E}=\mathrm{mc}^{\wedge} 2$, plug in the $\mathrm{eV}$ (electron volts) for whichever fermion or boson you choose. Let's say gravitation has a relative strength of 1 . Physics then says electromagnetism is $10^{\wedge} 36$ (a trillion trillion trillion times stronger), the strong nuclear interaction is $10^{\wedge} 38$, and the weak nuclear interaction is $10^{\wedge} 25$. Then a photon multiplied by (combined with) $10^{\wedge} 2$ gravitons might result in that matter's strong force while a photon divided by (inversely combined with) $10^{\wedge} 11$ gravitons might result in that matter's weak force. 